\title{
Breit-Wigner Enhancement of Dark Matter Annihilation
}

\author{
Masahiro Ibe, ${ }^{1}$ Hitoshi Murayama, ${ }^{2,3,4}$ and T. T. Yanagida ${ }^{5,2}$ \\ ${ }^{1}$ SLAC National Accelerator Laboratory, Menlo Park, CA 94025 \\ ${ }^{2}$ Institute for the Physics and Mathematics of the Universe, University of Tokyo, Kashiwa 277-8568, Japan \\ ${ }^{3}$ Department of Physics, University of California, Berkeley, CA 94720 \\ ${ }^{4}$ Theoretical Physics Group, Lawrence Berkeley National Laboratory, Berkeley, CA 94720 \\ ${ }^{5}$ Department of Physics, University of Tokyo, Tokyo 113-0033, Japan.
}

(Dated: October 29, 2018)

\begin{abstract}
We point out that annihilation of dark matter in the galactic halo can be enhanced relative to that in the early universe due to a Breit-Wigner tail, if the dark matter annihilates through a pole just below the threshold. This provides a new explanation to the "boost factor" which is suggested by the recent data of the PAMELA, ATIC and PPB-BETS cosmic-ray experiments.
\end{abstract}

Dark matter of the universe has been discussed since 1933, yet its nature still remains elusive [1]. Seventy-five years later we only managed to restrict its mass between $10^{-31}$ and $10^{50} \mathrm{GeV}$, demonstrating our lack of understanding. However, the thermal relic of a Weakly Interacting Massive Particle (WIMP) is arguably best theoretically motivated because it has the same mass scale as the anticipated new physics that would explain why our universe is a superconductor (electroweak symmetry breaking). Hopes are high to discover WIMPs at the forthcoming LHC experiments, to detect them directly in sensitive underground experiments, as well as to observe signals of WIMP annihilations from the galactic center or the halo in high-energy cosmic rays.

Recent observations of the PAMELA [2], ATIC [3], and PPB-BETS 4] experiments strongly suggest the existence of a new source of positrons (and electrons) in cosmic rays. The most interesting interpretation of these results is the annihilation of the dark matter with a mass at the $\mathrm{TeV}$ scale. However, such interpretation requires that the annihilation cross section of the dark matter in the galactic halo is much larger (by a factor of $O(100)$ ) than the one appropriate to explain the dark matter relic density precisely measured by the WMAP experiment [5].

The enhancement of the dark matter annihilation in the galactic halo is called a "boost factor." So far, there have been several proposals to explain the origin of the boost factor both from astrophysics such as the enhanced local dark matter density, and from particle physics such as the Sommerfeld enhancement due to an attractive force among the dark matter particles $[6]$.

In this letter, we propose a new explanation of the boost factor. We consider the dark matter which annihilates via a narrow Breit-Wigner resonance just below the threshold. When the resonance mass is just below twice the dark matter mass, the annihilation cross section becomes sensitive to the velocity of the dark matter. In such a case, the time evolution of the dark matter abundance is quite different from the one in the usual non-resonant case of annihilation, and we find that the annihilation cross section in the halo is enhanced compared to what is inferred from the relic abundance. As we will show, the cross section required from the dark matter density can be large enough to explain the PAMELA, ATIC, and PPB-BETS results, and hence, we do not need in our proposal any additional boost factor due to an overdense region in the halo or the Sommerfeld enhancement.

Cross Section Just Above a Pole. In this study, we assume that the dark matter with mass $m$ annihilates via a narrow resonance $R$. For a simplicity, we consider a scalar resonance, although generalization to arbitrary spins is straight forward. The general formula for the scattering cross section via a resonance $R$ is given by

$$
\sigma=\frac{16 \pi}{E_{\mathrm{cm}}^{2} \bar{\beta}_{i} \beta_{i}} \frac{M^{2} \Gamma^{2}}{\left(E_{\mathrm{cm}}^{2}-M^{2}\right)^{2}+M^{2} \Gamma^{2}} B_{i} B_{f},
$$

where $M$ and $\Gamma$ are the mass and the decay rate of the resonance $R$, respectively. Two body initial and final states are assumed and $\bar{\beta}_{i}=\sqrt{1-4 m^{2} / M^{2}}$ is the initial state phase space factor evaluated on the resonance while $\beta_{i}=\sqrt{1-4 m^{2} / E_{\mathrm{cm}}^{2}}$ at the center of mass energy of the collision. The branching fractions of the resonance into the initial and final states are denoted by $B_{i}$ and $B_{f}$, respectively. Note that since we are assuming an unphysical pole, i.e., $2 m>M, B_{i}$ and $\bar{\beta}_{i}$ are not physical and should be understood as analytic continuations of those quantities from the physical region, $2 m<M$. Even so, a combination $B_{i} / \bar{\beta}_{i}$ is well-defined in both regions, and hence, the above cross section is also well-defined even in the unphysical pole case.

The dark matter annihilation in the early universe must be thermally averaged. On the other hand, the dark matter annihilation in the galactic halo is averaged over the velocity distribution, which can be approximated by the Maxwellian distribution. In both cases, the dark matter is non-relativistic. Therefore, in either case, we can use the Gaussian average,

$$
\left\langle\sigma v_{\text {rel }}\right\rangle=\frac{1}{\left(2 \pi v_{0}^{2}\right)^{3}} \int d \vec{v}_{1} d \vec{v}_{2} e^{-\left(\vec{v}_{1}^{2}+\vec{v}_{2}^{2}\right) / 2 v_{0}^{2}} 2 \sigma \beta_{i},
$$

where $\vec{v}_{1,2}$ are the velocities of the initial states and we have used $v_{\text {rel }}=2 \beta_{i}$. The non-relativistic approximation gives

$$
E_{\mathrm{cm}}^{2}=4 m^{2}+m^{2}\left(\vec{v}_{\mathrm{rel}}\right)^{2}, \quad\left(\vec{v}_{\mathrm{rel}}=\vec{v}_{1}-\vec{v}_{2}\right) .
$$


Now, let us consider the annihilation process near a narrow resonance, i.e.,

$$
M^{2}=4 m^{2}(1-\delta), \quad|\delta| \ll 1 .
$$

Note that positive $\delta$ implies that the pole is just below the threshold of the dark matter annihilation. With this notation, we can rewrite the above cross section as,

$$
\sigma=\frac{16 \pi}{M^{2} \bar{\beta}_{i} \beta_{i}} \frac{\gamma^{2}}{\left(\delta+\vec{v}_{\mathrm{rel}}^{2} / 4\right)^{2}+\gamma^{2}} B_{i} B_{f}
$$

where we have defined,

$$
\gamma=\Gamma / M
$$

Furthermore, we have verified that we can approximate the Gaussian integral reasonably well by

$$
\left\langle\sigma v_{\mathrm{rel}}\right\rangle \simeq \frac{32 \pi}{M^{2} \bar{\beta}_{i}} \frac{\gamma^{2}}{\left(\delta+\xi v_{0}^{2}\right)^{2}+\gamma^{2}} B_{i} B_{f}
$$

where a parameter $\xi \approx 1 / \sqrt{2}$ gives the best fit to the numerical results for $v_{0} \ll 1$ and $\delta>0$. This expression shows that the denominator is dominated by the $\vec{v}_{\text {rel }}^{2}$ term for $|\delta|, \gamma \ll \xi v_{0}^{2}$, while the other terms dominate when the velocity is much smaller. Therefore, the cross section is sensitive to the parameters $\delta$ and $\gamma$, and have an enhanced behavior at the lower temperature for small $\delta$ and $\gamma$. This main point of this letter can be seen easily in a schematic plot in Fig. 1.

Note that the approximation given in Eq. (7) is not a good one for the dark matter with a rather large velocity, i.e., $v_{0} \gtrsim 0.1[7]$. The approximation also becomes worse around the pole in the physical region, possible if $\delta<0$. In the following analysis, we mainly consider the unphysical pole $\delta>0$. As we will see below, for this case, the resultant dark matter density is mostly determined by the dynamics of the dark matter at $v_{0} \ll 1$, and hence the approximated cross section works quite well. We will later also briefly discuss the case of a physical pole, $\delta<0$.

Time Evolution of Dark Matter Density. Now let us consider the time evolution of the relic density of the dark matter. The most notable feature of our proposal is that the annihilation process does not freeze out even after the "freeze-out" time for the usual non-resonant annihilation models. That is, the interaction rate can be larger than the Hubble expansion rate even after a "freeze-out" time, since the cross section is enhanced at the lower temperature. In the following, to avoid confusion, we define the "freeze-out" time $\tilde{x}_{f}$ by the usual meaning at which the yield of the dark matter $Y=n / s$ starts deviating from the value in the thermal equilibrium, i.e., $Y-Y_{\mathrm{EQ}}=O\left(Y_{\mathrm{EQ}}\right)$. As we will show, the actual freezeout time is much later than $\tilde{x}_{f}$. In this section, we mainly consider the unphysical pole, $\delta>0$.

Following Ref. [8], section 5.2, we write down the Boltzmann equation of the yield of the dark matter [10],

$$
\frac{d Y}{d x}=-\frac{\lambda}{x^{2}} \frac{\left(\delta^{2}+\gamma^{2}\right)}{\left(\delta+\xi x^{-1}\right)^{2}+\gamma^{2}}\left(Y^{2}-Y_{\mathrm{EQ}}^{2}\right) .
$$

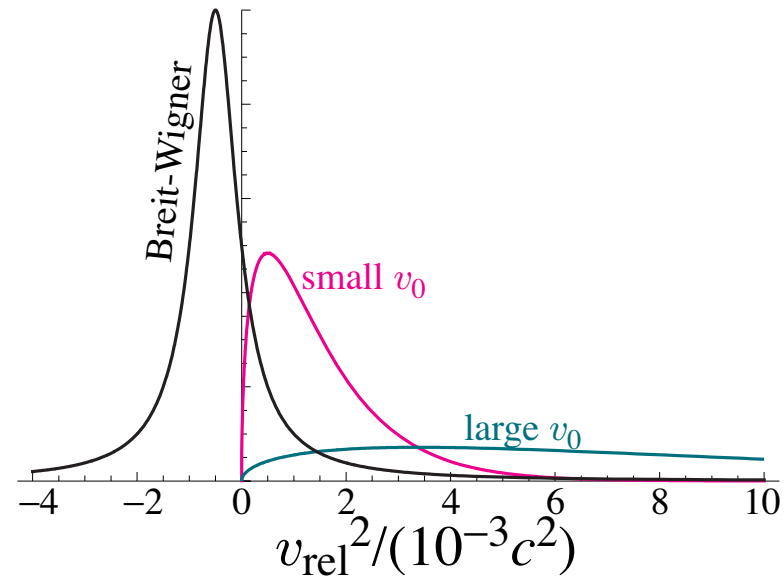

FIG. 1: A schematic plot that shows dispersion in relative velocity $v_{\text {rel }}^{2}$ and an unphysical pole in the cross section at $v_{\text {rel }}^{2}<0$ (below threshold). It is clear that a smaller dispersion $v_{0}$ gives a larger overlap with the Breit-Wigner tail in the cross section and hence an enhanced averaged cross section.

Here, we have used the following definitions

$$
\begin{aligned}
\lambda & =\left[\frac{s(T)}{H(T)}\right]_{T=m}\left\langle\sigma v_{\mathrm{rel}}\right\rangle_{T=0}=\sqrt{\frac{8 \pi^{2}}{45} g_{*}} M_{\mathrm{PL}} m \sigma_{0}, \\
\sigma_{0} & =\left\langle\sigma v_{\mathrm{rel}}\right\rangle_{T=0}=\frac{32 \pi B_{i} B_{f}}{M^{2} \bar{\beta}_{i}} \frac{\gamma^{2}}{\delta^{2}+\gamma^{2}}, \\
Y_{\mathrm{EQ}} & =\frac{45}{4 \sqrt{2} \pi^{7 / 2}}\left(\frac{g_{i}}{g_{*}}\right) x^{3 / 2} e^{-x}, \\
x & =\frac{m}{T}=v_{0}^{-2} .
\end{aligned}
$$

The parameter $g_{*}\left(g_{i}\right)$ is the number of the degrees of freedom for massless particles (dark matter), respectively. Note that we have used the reduced Planck scale $M_{\mathrm{PL}} \simeq 2.4 \times 10^{18} \mathrm{GeV}$.

As we have defined, the "freeze-out" time $\tilde{x}_{f}$ is determined by $Y-Y_{\mathrm{EQ}}=O\left(Y_{\mathrm{EQ}}\right)$, and hence, the value of $\tilde{x}_{f}$ is not so sensitive to the parameters $\delta$ and $\gamma$ and mainly determined by the exponential suppression factor in $Y_{\mathrm{EQ}}$. Thus, we can expect that the value of $\tilde{x}_{f}$ is comparable to the freeze-out temperature $x_{f}$ in the usual non-resonant annihilation models, i.e., $\tilde{x}_{f} \approx x_{f} \approx O(10)$.

Unlike the non-resonant case where the annihilation cross section stays constant $\left\langle\sigma v_{\text {rel }}\right\rangle$ once non-relativistic, however, the annihilation cross section here increases as temperature drops. As a result, the annihilation process does not freeze out even for $x>\tilde{x}_{f}$ and the dark matter keeps annihilating until the temperature comes down to

$$
x_{b} \simeq \xi^{-1} \times \max [\delta, \gamma]^{-1} \gg \tilde{x}_{f} .
$$

Below this temperature, Eq. (8) reduces to

$$
\frac{d Y}{d x}=-\frac{\lambda}{x^{2}} Y^{2}
$$


and we obtain an asymptotic solution,

$$
Y_{\infty} \simeq \frac{1}{\lambda} x_{b} \simeq \frac{1}{\lambda} \times \max [\delta, \gamma]^{-1} .
$$

In the Fig.2, we show the time evolution of the yield $Y$ of the dark matter for a given parameter set. Here, we have used the numerical result of the Gaussian average of the cross section [11]. We also show the time evolution of the yield with the approximate cross section given in Eq. (7), $Y_{\text {app. As }}$ we have expected, the yield deviates from $Y_{\mathrm{EQ}}$ at $\tilde{x}_{f}=O(10)$, while the actual freeze-out occurs at $x_{b} \gg \tilde{x}_{f}$. From the figure, we see that $Y<Y_{\text {app }}$ during $\tilde{x}_{f}<x<x_{b}$. This means that the averaged cross section at $\tilde{x}_{f}$ is somewhat larger than the approximate one, while the final result is determined by the late time dynamics where the approximate cross section works well. The figure shows that the above approximate asymptotic solution gives a good estimate of the resultant yield of the dark matter.

It is worth comparing the asymptotic solution in Eq. (12) with the asymptotic solution in the usual nonresonant ( $S$-wave) annihilation models [8],

$$
Y_{\infty} \simeq \frac{1}{\lambda} x_{f}
$$

with $x_{f}=O(10)$. These two solutions show that the dark matter abundance in our proposal is larger by a factor

$$
\frac{x_{b}}{x_{f}} \simeq \frac{\max [\delta, \gamma]^{-1}}{O(10)}
$$

when we assume the same cross section at the zero temperature for both models. In the Fig.2, we have showed the time evolution of the yield in the usual non-resonant annihilation models assuming the same cross section at the zero temperature $\sigma_{0}$ used in $Y$ (i.e., $\lambda=10^{9}$ ). As expected, the yield in the non-resonant annihilation is more suppressed compared to the resonant annihilation. The physical reasons of this enhancement are as follows. First, in our proposal, the cross section is suppressed by $\left(\tilde{x}_{f} / x_{b}\right)^{2}$ at $\tilde{x}_{f}$, which results in a relatively larger abundance in a period of $x>\tilde{x}_{f}$. Second, the annihilation process is relatively less effective during $\tilde{x}_{f}<x<x_{b}$ compared to the usual annihilation case, although the annihilation does not freeze out in that period.

Before closing this section, we mention the model with a physical pole, i.e., $\delta<0$. In this case, the cross section given in Eq. (7) poorly approximates the thermal averaged cross section [7]. Especially, the thermal average can pick up the cross section at the pole, $v_{\text {rel }}^{2}=4|\delta|$ when the temperature is rather high, i.e., $x^{-1} \gg|\delta|$. Thus, the averaged cross section can be much higher than that expected in the unphysical pole where the cross section is suppressed by $x^{2}\left(\delta^{2}+\gamma^{2}\right)$ at high temperature. Thus, the annihilation cross section at $\tilde{x}_{f}$, which typically satisfies

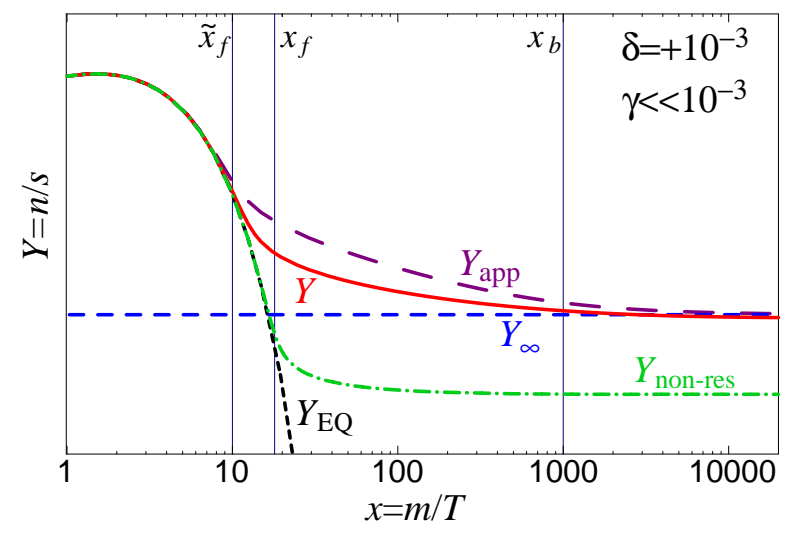

FIG. 2: The time evolution of the yield $Y$ of the dark matter in terms of the parameter $x=m / T$ for given values of $\delta$ and $\gamma$ (the solid line). In this figure, we assume $\delta>0$ and the pole is not in the physical region. The long-dashed line labeled $Y_{\text {app }}$ is the evolution with the approximated cross section in Eq. (7). The dashed line labeled $Y_{\infty}$ is the asymptotic solution $Y_{\infty}$ given in Eq. (12). The short-dashed line represents the equilibrium yield $Y_{\mathrm{EQ}}$. The dash-dotted line labeled $Y_{\text {non-res }}$ shows the time evolution of the yield in the usual (non-resonant) freeze-out assuming the same cross section at the low temperature $\sigma_{0}$ used in $Y$ (see Eq. (13)). The boost factor is the asymptotic value of $Y / Y_{\text {non-res }}$.

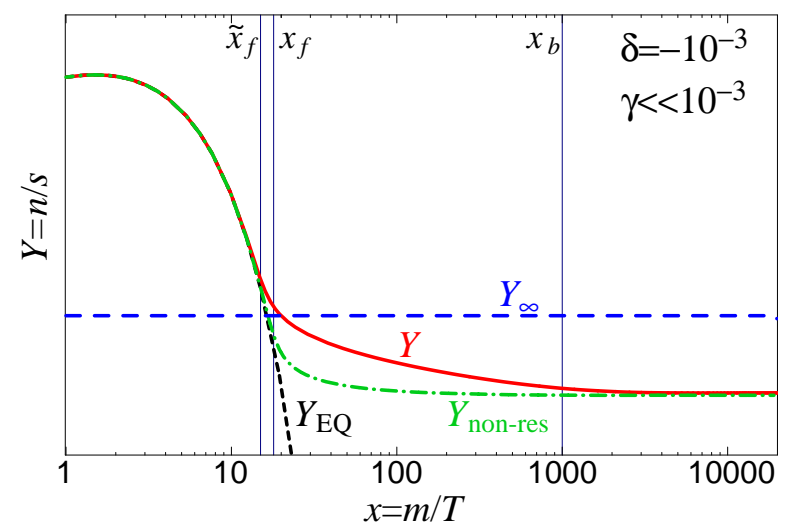

FIG. 3: The time evolution of the yield of the dark matter $Y$ for $\delta<0$ (the solid line). Everything else is the same as in Fig. 2. There is practically no boost for this parameter set.

$\tilde{x}_{f}^{-1} \gg|\delta|, \gamma$, is much larger than that in the case of the unphysical pole. Therefore, the yield enhancement seen in the unphysical pole is much smaller in this case.

Effective Boost Factor. Finally, let us work out the boost factor for annihilation in the galactic halo. As we have seen, the dark matter abundance is enhanced by a factor of $\left(x_{b} / x_{f}\right)$ for $\delta>0$ compared to the usual non-resonant annihilation models for a given annihilation cross section at the zero temperature, $\sigma_{0}$. This means that the cross section $\sigma_{0}$ must be larger than the one 
expected in the usual models to reproduce the observed dark matter density.

More explicitly, the yield given in Eq. (12) is translated to the mass density parameter,

$$
\Omega_{\mathrm{DM}} h^{2} \simeq 0.1 \times\left(\frac{10^{-9} \mathrm{GeV}^{-2}}{\sigma_{0}}\right)\left(\frac{x_{b}}{x_{f}}\right),
$$

where we have used $x_{f} \simeq 20$ and $g_{*} \simeq 200$. Therefore, the observed dark matter density $\Omega h^{2} \simeq 0.1$ requires

$$
\sigma_{0} \simeq 10^{-9} \mathrm{GeV}^{-2} \times\left(\frac{x_{b}}{x_{f}}\right),
$$

which is much larger than what is expected in the usual annihilation case, i.e.,

$$
\sigma_{0} \simeq 10^{-9} \mathrm{GeV}^{-2}
$$

In the galactic halo, the average velocity is given by $v_{0} \simeq 10^{-3}$, and the cross section is well approximated by the one at the zero temperature as long as $v_{0}^{2} \ll \delta, \gamma$. Thus, we can achieve the large annihilation cross section suggested by the PAMELA, PPB-BETS, and ATIC anomalies, $\left\langle\sigma v_{\text {rel }}\right\rangle_{T=0}=O\left(10^{-(6-7)}\right) \mathrm{GeV}^{-2}$ for $\delta, \gamma \lesssim 10^{-3}$. Therefore, in our proposal, we can explain the large annihilation cross section in the galactic halo without other boost factors.

For convenience, we could define an effective boost factor as the ratio between the cross sections in the usual and our models. From Eqs. (16) and (17) we obtain

$$
\mathrm{BF} \simeq \frac{x_{b}}{x_{f}} \simeq \frac{\max [\delta, \gamma]^{-1}}{x_{f}} \simeq \frac{\max [\delta, \gamma]^{-1}}{O(10)}
$$

In this way, we can explain the boost factor of $O(100)$, in a model with $\delta, \gamma=O\left(10^{-3}\right)$, as seen in Fig. 4 .

Discussion. In this letter, we showed that the boost factor required in recent observations of cosmic ray electrons and positrons can be obtained if the dark matter annihilates via a narrow resonance just below the threshold. Because the annihilation in the early universe is suppressed by the Breit-Wigner tail, the observed dark matter density requires a larger-than-normal cross section which can be consistent with the PAMELA, ATIC and PPB-BETS results. The required cross section is achieved for $4 m^{2}-M^{2}, M \Gamma \lesssim 10^{-3} M^{2}$.

Note that the small decay width $\Gamma \ll 10^{-3} M$ can be achieved rather easily, when the resonance $R$ is a weakly coupled particle. In some models, a coincidence in masses can also be naturally realized. For example, if the dark matter is the lightest Kaluza-Klein (KK) particle and the resonance is at the second $\mathrm{KK}$ level, we have the relation $M=2 m$ at the tree-level [9] [12] (see also a recent discussion in Ref. [3]). Thus, in such models, we may well have a small mass splitting $\delta$ as a result of small radiative corrections to the tree-level mass relation.

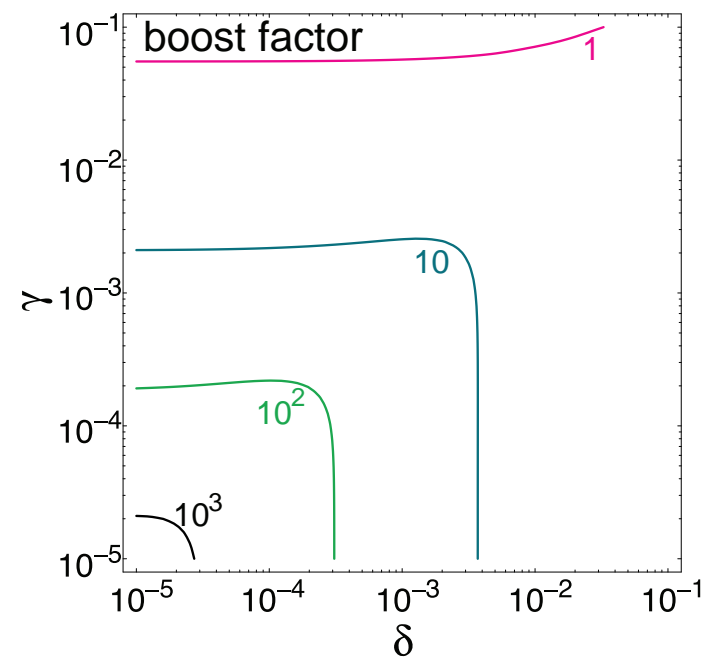

FIG. 4: The boost factor on the $(\delta, \gamma)$ plane. Thermal average is done numerically without relying on the approximation Eq. (7).

The work of M.I. was supported by the U.S. Department of Energy under contract number DE-AC0276SF00515. The work of H.M. and T.T.Y. was supported in part by World Premier International Research Center Initiative (WPI Initiative), MEXT, Japan. The work of H.M. was also supported in part by the U.S. DOE under Contract DE-AC03-76SF00098, and in part by the NSF under grant PHY-04-57315.

[1] See, e.g., H. Murayama, arXiv:0704.2276 [hep-ph].

[2] O. Adriani et al., arXiv:0810.4995 [astro-ph].

[3] J. Chang et al., Nature 456, 362 (2008).

[4] S. Torii et al., arXiv:0809.0760 [astro-ph].

[5] E. Komatsu et al. [WMAP Collaboration], arXiv:0803.0547 [astro-ph].

[6] N. Arkani-Hamed, D. P. Finkbeiner, T. Slatyer and N. Weiner, arXiv:0810.0713 [hep-ph].

[7] K. Griest and D. Seckel, Phys. Rev. D 43, 3191 (1991).

[8] E. W. Kolb and M. S. Turner, "The Early Universe," Frontiers in Physics, Westview Press, 1994.

[9] M. Kakizaki, S. Matsumoto, Y. Sato and M. Senami, Phys. Rev. D 71, 123522 (2005) arXiv:hep-ph/0502059.

[10] The analysis of the Boltzmann equation near a resonance has been developed in Ref. [7].

[11] In the computation, we have used $\lambda=10^{9}$. The qualitative behavior of the time evolution does not depend on $\lambda$, although the values of $x_{f}$ and $\tilde{x}_{f}$ slightly depend on $\lambda$.

[12] In the explicit model given in the reference, the resonance mainly decays into a top pair which is not favored by the null results of the excess of the anti-proton flux [2, 3, 4]. 